\title{
Comparaison du peuplement fogique du rumen, du duodénum et des fèces de mouton
}

\author{
A Breton, I Confesson, M Dusser, B Gaillard-Martinie
}

\section{INRA, laboratoire de microbiologie, 63122 Saint-Genès-Champanelle, France}

Les champignons anaérobies stricts du rumen sont désormais bien connus pour leur rôle dans la colonisation et la dégradation des particules alimentaires. Peu de travaux concernent leur devenir après passage du rumen dans la caillette acide, l'intestin et enfin le milieu extérieur auquel ils accèdent par le biais des fèces. En conséquence, on ignore si la flore fongique est modifiée, qualitativement et quantitativement, au cours de ce transit.

Les dénombrements ont été réalisés par la technique des roll-tubes de Joblin (1981) à partir de contenu ruminal et duodénal et de fèces prélevés $1 \mathrm{~h}$ après le repas dans un mouton fistulisé. La colonisation des particules a été observée en microscopie de fluorescence après lavage dans du tampon PBS et contact avec la lectine de blé (WGA) marquée à l'isothiocyanate de fluorescéine spécifique du chitobiose des parois fongiques.

Sur plusieurs mois, le niveau du peuplement fongique varie de 8500 à 45600 unités formant une colonie (UFC) par $\mathrm{ml}^{-1}$ de contenu ruminal et de 320 à 1675 UFC g-1 de fèces. Les analyses de contenu duodénal se sont toujours révélées négatives. Dans le rumen, $70 \%$ des champignons sont de type filamenteux monocentrique ( $N e O-$ callimastix, Piromyces), $27 \%$ de type vésiculeux (Caecomyces) et 3\% comportent une thalle polycentrique (Orpinomyces). En revanche, dans les fèces les Caecomyces prédominent $(75 \%)$, les polycentriques sont absents, et les monocentriques filamenteux (25\%) sont en majorité des Piromyces parmi lesquels $\boldsymbol{P}$ mae a été identifié.

En microscopie de fluorescence, les particules alimentaires du rumen apparaissent plus ou moins colonisées ; tous les stades de développement des thalles sont présents. Au niveau du duodénum, de gros sporocystes non viables fixés

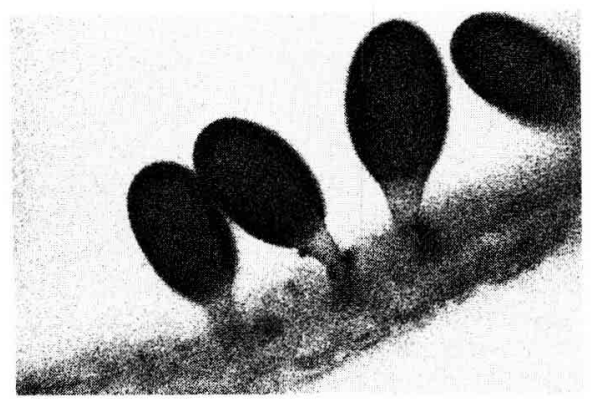

Fig 1. Sporocystes sur particules dans le duodénum. Microscopie de fluorescence avec WGA (x 500).

aux particules se singularisent par une paroi très épaisse. Dans le matériel fécal, les particules sont peu colonisées par des petites structures globuleuses de $10 \mu$ en moyenne, correspondant à des spores enkystées qui assurent, dans les fèces deshydratées, la survie des champignons pendant au moins 150 j avec une perte de viabilité de $40 \%$.

En conclusion, la flore fongique des fèces de mouton, comme pour la vache (Grenet et al, 1989), se distingue par rapport à celle du rumen par un peuplement réduit composé essentiellement de Caecomyces et Piromyces. Ces champignons, qui n'ont pû être isolés du duodénum, constituent une flore autochtone intestinale dont l'origine est à préciser.

Joblin KN (1981) Appl Environ Microbiol 42, 11191122

Grenet E, Fonty G, Jamet J, Bonnemoy F (1989) Appl Environ Microbiol 55, 2360-2364 